IJMMS 26:12 (2001) 765-770

PII. S0161171201010729

http://ijmms.hindawi.com

(c) Hindawi Publishing Corp.

\title{
FREE OBJECTS IN THE CATEGORY OF GEOMETRIES
}

\author{
TALAL ALI AL-HAWARY
}

(Received 15 August 2000)

\begin{abstract}
The aim of this note is to introduce the class of free geometries purely in terms of morphisms. Several classes of well-known matroid morphisms are characterized via the new concept.
\end{abstract}

2000 Mathematics Subject Classification. 05B35.

1. Introduction. We shall assume familiarity with category and matroid theories; for an introduction, see [2, 3], respectively. In particular, a matroid $M$ is an ordered pair $\left(E, \mathfrak{f}_{M}\right)$ where $\mathfrak{f}_{M}$ is a collection of subsets, called flats of $M$, of a finite set $E$ such that $E$ is a flat of $M$, the intersection of any two flats of $M$ is a flat of $M$ and if $F \in \mathfrak{f}_{M}$ and $\left\{F_{1}, F_{2}, \ldots, F_{k}\right\}$ is the set of minimal members of $\mathfrak{f}_{M}$ (with respect to inclusion) that properly contain $F$ (denoted by $F_{i} \succ F$ ), then $F_{1} \cup F_{2} \cup \cdots \cup F_{k}=E$. The set $E$ is called the ground set of $M$. A formal notation for the matroid on the ground set $E$ with flats $\mathfrak{f}_{M}$ is $M\left(E, \mathfrak{f}_{M}\right)$, but when no confusion will arise, we refer to this matroid as $M$. When several matroids $M\left(E_{i}, \mathfrak{f}_{i}\right), i=1,2, \ldots, n$ are being considered, we shall often denote these matroids by $M_{1}, M_{2}, \ldots, M_{n}$.

By a combinatorial geometry we mean a loopless matroid with no multiple elements, that is, a matroid in which the empty set and each point, if any exists, is a flat. We will use the shorter "geometry" in place of "combinatorial geometry." A free geometry is a geometry which has every subset of its ground set as a flat. If the ground set of a free geometry has $m$ elements, then we denote that geometry by $U_{m, m}$.

Our main goal in this note is to introduce a categorical definition of a free object in the category $\varphi$ of geometries and strong maps. We define a functor, which we call a free functor, from the subcategory $\mathscr{F}$ of free geometries to the category $\mathscr{G}$. Lastly, we show that $\mathscr{F}$ is a coreflective subcategory of $\mathscr{G}$ and the free functor is a faithful functor which is a right adjoint of the inclusion functor.

The category of free geometries play an important role in solving the following open problem:

Find a finite set of elementary axioms that characterize the category $\mathscr{G}$.

It was that problem which prompted us to study the notion of free geometries.

2. Free objects. Define the isthmus 1 to be an object in $\mathscr{G}$ with exactly one endomorphism. Since in any category with an object $M$, the identity map $i_{M}$ is always an endomorphism of $M, i_{1}$ is the endomorphism of 1 . Observe that 1 is the terminal object of $\mathscr{G}$ while the initial object 0 is the empty geometry which is isomorphic to $U_{0,0}$. 
Proposition 2.1. For every object $M, x$ is an element of $M$, or $x \in M$, if and only if $x$ is a morphism with $1 \stackrel{x}{\rightarrow} M$.

In the concrete category of geometries and strong maps, 1 is isomorphic to the free geometry $U_{1,1}$. Clearly $U_{1,1}$ has one element and one endomorphism. We shall denote the element of 1 by $c$. Next, we give a categorical definition of free objects which has never been introduced before. We remark that this definition is not obvious.

DEFINITION 2.2. An object $D$ is called a free object if for every $x \in D$ there exists a morphism $h_{x}$ with $D \stackrel{h_{x}}{\longrightarrow} 1 \amalg 1$ such that for every $y \in D, y \neq x$, we have $h_{x} x \neq h_{x} y$.

Clearly the objects 0,1 are free. Also $1 \amalg 1$ is free since $1 \amalg 1$ has only two elements and the identity on $1 \amalg 1$ satisfies the property of $h_{x}$ in the definition of free objects. We notice as 1 is isomorphic to $U_{1,1}$ that has a ground set isomorphic to $\{c\}$, the geometry $1 \amalg 1$ has a ground set isomorphic to $\left\{c_{1}, c_{2}\right\}$. Next, we state and prove our first main result.

THEOREM 2.3. A geometry is free if and only if it is isomorphic to $U_{n, n}$.

Proof. Let $D=M\left(E, \mathfrak{f}_{D}\right)$ be a free geometry such that $|E|=n$. If $n=0$, then $D \cong U_{0,0}$. If $n=1$, then $D \cong 1 \cong U_{1,1}$. If $n \geq 2$, then to show $D \cong U_{n, n}$ it is sufficient to show that $\{x\}$ and $E \backslash\{x\}$ are flats of $D$ for all $x \in E$. Since then, for every proper subset $F \subset E$ such that $|F| \leq n-1, F \in \mathfrak{f}_{D}$. Let $x \in E$. Then the constant map $f_{x}$ with $1 \stackrel{f_{x}}{\longrightarrow} D$ where $f_{x}(c)=x$ is a strong map. Hence as $D$ is free, there exists a strong map $h_{x}$ with $M \stackrel{h_{x}}{\longrightarrow} 1 \amalg 1$ such that for every strong map $g$ with $1 \stackrel{g}{\rightarrow} M, g \neq f_{x}$, we must have $h_{x} f_{x} \neq h_{x} g$. Thus assume $h_{x} f(c)=c_{1}$. Again as $D$ is free and as for all $y \in E \backslash\{x\}$, the constant map $f_{y}$ as above is a strong map such that $f_{y} \neq f_{x}$, we have $h_{x} f_{x}(c) \neq h_{x} f_{y}(c)$. Thus, $h_{x}(y)=c_{2}$ for all $y \in E \backslash\{x\}$. As $\left\{c_{1}\right\}$ and $\left\{c_{2}\right\}$ are flats of $1 \amalg 1,\{x\}=h_{x}^{-1}\left(\left\{c_{1}\right\}\right)$ and $E \backslash\{x\}=h_{x}^{-1}\left(\left\{c_{2}\right\}\right)$ are flats of $D$. Therefore, $D \cong U_{n, n}$.

If $D \cong U_{n, n}$ for some $n$ where $U_{n, n}$ has a ground set $E$, then for every strong map $f$ with $1 \stackrel{f}{\rightarrow} D$, define a strong map $h$ with $U_{n, n} \stackrel{h}{\rightarrow} 1 \amalg 1$ by $h(z)=c_{1}$ when $z=f(c)$, and $h(z)=c_{2}$ otherwise. For every strong map $g$ with $1 \stackrel{g}{\rightarrow} M$ such that $g \neq f, g(c) \neq f(c)$ and hence $h g \neq h f$. Therefore, $D$ is free.

The proof of the following weak axiom of choice follows directly from the axiom of choice for sets.

THEOREM 2.4. For every morphism $f$ with $M_{1} \stackrel{f}{\rightarrow} D$ where $M_{1} \supsetneqq 0$ and $D$ is a free object, there exists a morphism $g$ with $D \stackrel{g}{\rightarrow} M_{1}$ such that $f=f g f$.

Next, we show 1 is a generator and use that to give a sufficient condition for a morphism in the category $\mathscr{G}$ to be an epimorphism.

LEMMA 2.5. The isthmus object 1 is a generator.

Proof. If $M$ and $N$ are objects and $f, g$ are morphisms from $M$ to $N$ such that $f \neq g$, then there exists $x \in M$ (i.e., $x$ is a morphism with $1 \stackrel{x}{\rightarrow} M$ ) such that $f x \neq g x$. Thus 1 is a generator. 


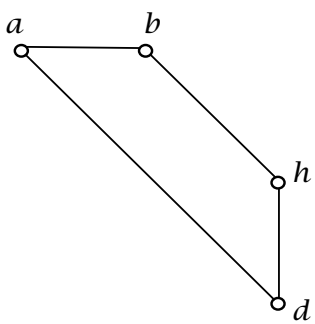

$M$

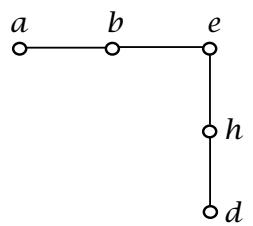

$N$

FIGURE 2.1. The converse of Proposition 2.5 does not hold.

PROPOSITION 2.6. If $M_{1}$ and $M_{2}$ are two objects and $f$ is a morphism with $M_{1} \stackrel{f}{\rightarrow} M_{2}$ such that for every element $g \in M_{2}$ there exists an element $h \in M_{1}$ satisfying $g=f h$, then $f$ is an epimorphism.

Proof. If $N$ is an object and $f_{1}, f_{2}$ are two morphisms with $M_{2} \stackrel{f_{1}}{\longrightarrow} N$ and $M_{2} \stackrel{f_{2}}{\longrightarrow} N$ such that $f_{1} f=f_{2} f$, then we need only show the right cancellation law holds, that is, $f_{1}=f_{2}$. Suppose $f_{1} \neq f_{2}$. By Lemma 2.5, 1 is a generator and hence there exists a morphism $m$ with $1 \stackrel{m}{\rightarrow} M_{2}$ such that $f_{1} m \neq f_{2} m$. Thus by assumption, there exists a morphism $k$ with $1 \stackrel{k}{\rightarrow} M_{1}$ such that $m=f k$ and hence, $f_{1} f k=f_{1} m \neq f_{2} m=f_{2} f k$. That is, $f_{1} f k \neq f_{2} f k$ which is a contradiction to the fact that $f_{1} f h=f_{2} f h$ (because $\left.f_{1} f=f_{2} f\right)$.

Next we show the converse of the preceding proposition need not hold in $\mathscr{G}$.

EXAMPLE 2.7. Consider the matroids $M$ and $N$ given by the point configurations in Figure 2.1. By [1, Proposition 3], the inclusion map $i$ with $M \stackrel{i}{\rightarrow} N$ is an epimorphism. Define a strong map $f$ with $1 \stackrel{f}{\rightarrow} N$ by $f(c)=e$. If $g$ is a strong map with $1 \stackrel{g}{\rightarrow} M$ such that $f=i g$, then $g(c)=i g(c)=f(c)=e$ which is a contradiction to the fact that $e \notin E(M)$. Therefore, the converse of Proposition 2.6 does not hold.

3. Some peculiar morphisms. In [1], Crapo proved that a strong map is a monomorphism if and only if it is a one to one map on points. It was also shown that an onto strong map, on points, is an epimorphism but an epimorphism need not be onto, on points. Next, we show that an epimorphism with free codomain is onto, on points.

Proposition 3.1. If $f$ with $M \stackrel{f}{\rightarrow} D$ is an epimorphism where $D$ is free, then $f$ is an onto map on points.

Proof. Suppose $E(M)$ and $E(D)$ are the ground sets of $M$ and $D$, respectively. If $f$ is not onto, then there exists $x \in E(D)$ such that $x \notin f(E(M))$. Let $H$ be a geometry on the set $\{x, y\}$ and define strong maps $g$ and $h$ from $D$ to $H$ by $g(x)=y, g(z)=x$ when $z \in f(E(M))$ and $h(z)=x$ for all $z \in E(D)$. Then $g f=h f$ and $g \neq h$. Therefore, $f$ is not an epimorphism.

Proposition 3.2. Every nonzero object $M$ has elements and the morphism $t$ with $M \stackrel{t}{\rightarrow} 1$ is an epimorphism. 
Proof. By Theorem 2.4, there exists a morphism $h$ with $1 \stackrel{h}{\rightarrow} M$ such that $t=t h t$ and hence $t h=t h t h$. Therefore, $t h=i_{1}$ and as $i_{1}$ is the only endomorphism of 1 , by Proposition 2.6, $t$ is an epimorphism.

Proposition 3.3. Any bimorphism (= a monomorphism and an epimorphism) $f$ with $M_{1} \stackrel{f}{\rightarrow} M_{2}$ with free domain and codomain is an isomorphism.

PROoF. If $M_{1} \cong 0$, by Proposition $3.2, M_{2} \cong 0$ since $f$ is an epimorphism. If $M_{1} \supsetneqq 0$, by Theorem 2.4, there exists a morphism $g$ with $M_{2} \stackrel{g}{\rightarrow} M_{1}$ such that $f=f g f$ and since $f$ is a bimorphism $g f=i_{M_{1}}$ and $f g=i_{M_{2}}$. Thus $f$ is an isomorphism.

The following theorem indicates that the category of free objects and strong maps is a coreflective subcategory of $\mathscr{G}$. The proof of that theorem is not hard and is thus left to the reader.

THEOREM 3.4. For every object $M$, there exists a free object $|M|$ together with a morphism $t_{M}$ with $|M| \stackrel{t_{M}}{\longrightarrow} M$ such that for every free object $D$ and a morphism $h$ with $D \stackrel{h}{\rightarrow} M$, there exists a unique morphism $k$ with $D \stackrel{k}{\rightarrow}|M|$ such that $h=t_{M} k$. That is to say, the subcategory $\mathscr{F}$ of free geometries and strong maps is a coreflective subcategory of $\varphi$.

Next, we state and prove several facts related to the morphisms $t_{M}$ and the objects $|M|$, all purely in terms of morphisms only.

Proposition 3.5. The morphism $t_{M}$ is a bimorphism.

Proof. By Theorem 3.4 and Proposition 2.6, $t_{M}$ is an epimorphism. If $x$ and $y$ are elements of $|M|$ such that $t_{M} x=t_{M} y$, then as 1 is a free object, by Theorem 3.4, there exists a unique element $h \in|M|$ such that $t_{M} x=t_{M} h$. But as $t_{M} y=t_{M} x, x=h=y$ and hence $t_{M}$ is a monomorphism.

Observe that $|M|$ is defined up to isomorphism and the operation " | " is a functor which we call the free functor. Next, we prove the free functor is a faithful functor that is also a right adjoint of the inclusion functor from $\mathscr{F}$ to $\mathscr{G}$.

Proposition 3.6. The free functor is faithful, that is, for every morphisms $f, g$ from $M$ to $N$ such that $|f|=|g|$, then $f=g$. Moreover, the free functor is a right adjoint of the inclusion functor $\mathscr{F} \hookrightarrow \mathscr{G}$.

Proof. We prove the first part of the proposition and leave the other to the reader. If $|f|=|g|$, then $f t_{M}=t_{N}|f|=t_{N}|g|=g t_{M}$ and since $t_{M}$ is an epimorphism, $f=g$.

The proof of the following proposition is immediate and is left to the reader.

Proposition 3.7. A morphism $f$ is a bimorphism if and only if $|f|$ is a bimorphism.

COROLLARY 3.8. If $f$ is a bimorphism with $D \stackrel{f}{\rightarrow} N$ where $D$ is a free object, then $D \cong|N|$. 


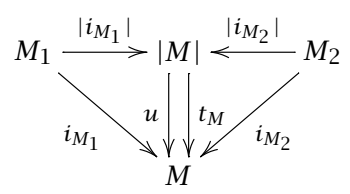

FIGURE 3.1. Coproduct of free objects is free.

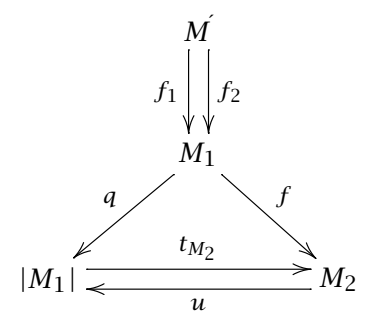

FIGURE 3.2. A regular epimorphism with free domain has a free codomain.

COROLLARY 3.9. If $f$ is a monomorphism with $M_{1} \stackrel{f}{\rightarrow} M_{2}$ and $M_{2}$ is a free object, then $M_{1}$ is a free object.

Proof. For every morphism $g$ with $1 \stackrel{g}{\rightarrow} M_{1}, f g$ is a morphism from 1 to $M_{2}$ and as $M_{2}$ is a free object, there exists a morphism $h_{f g}$ from $M_{2}$ to $1 \amalg 1$ such that $h_{f g} f g \neq$ $h_{f g} m$ for every morphism $m$ with $1 \stackrel{m}{\rightarrow} M_{2}$ such that $m \neq f g$. Let $e$ be a morphism with $1 \stackrel{e}{\rightarrow} M_{1}$ such that $e \neq g$. Then as $f$ is a monomorphism, $f e \neq f g$. Thus $h_{f g} f e \neq h_{f g} f g$. Therefore, $h f \neq h g$ where $h=h_{f g} f$ and hence $M_{1}$ is a free object.

COROLLARY 3.10. If $M_{1}$ and $M_{2}$ are free objects, then $M_{1} \amalg M_{2}$ is a free object.

Proof. As $M_{1}$ and $M_{2}$ are free objects, $M_{1} \cong\left|M_{1}\right|$ and $M_{2} \cong\left|M_{2}\right|$. By definition of the coproduct $M_{1} \amalg M_{2}$, there exists a unique morphism $u$ with $M \stackrel{u}{\rightarrow}|M|$, where $M \cong$ $M_{1} \amalg M_{2}$, such that $\left|i_{M_{1}}\right|=u i_{M_{1}}$ and $\left|i_{M_{2}}\right|=u i_{M_{2}}$. (See the diagram in Figure 3.1.) Thus $t_{M} u i_{M_{1}}=t_{M}\left|i_{M_{1}}\right|$ and $t_{M} u i_{M_{2}}=t_{M}\left|i_{M_{2}}\right|$. But by definition of $\left|i_{M_{1}}\right|$ and $\left|i_{M_{2}}\right|$, we have $t_{M}\left|i_{M_{1}}\right|=i_{M_{1}}$ and $t_{M}\left|i_{M_{2}}\right|=i_{M_{2}}$. Therefore, $i_{M}=t_{M} u$ and then as $i_{M}$ is a monomorphism, $u$ is a monomorphism. Thus since $|M|$ is a free object, by Corollary 3.9, $M$ is a free object.

COROLLARY 3.11. A regular epimorphism with a free domain has a free codomain.

PRoof. If $f$ is a morphism with $M_{1} \stackrel{f}{\rightarrow} M_{2}$ where $M_{1}$ is a free object, $M$ is an object and $f_{1}, f_{2}$ are two morphisms from $M$ to $M_{1}$ such that $\left\langle M_{1}, f\right\rangle$ is isomorphic to the coequalizer Coeq $\left(f_{1}, f_{2}\right)$ of $f_{1}$ and $f_{2}$, then by Theorem 3.4 , there exists a unique morphism $q$ with $M_{1} \stackrel{q}{\rightarrow}\left|M_{2}\right|$ such that $f=t_{M_{2}} q$. (See the diagram in Figure 3.2.) Thus, $t_{M_{2}} q f_{1}=f f_{1}=f f_{2}=t_{M_{2}} q f_{2}$ and since $t_{M_{2}}$ is a monomorphism, $q f_{1}=q f_{2}$. By definition of the coequalizer $\operatorname{Coeq}\left(f_{1}, f_{2}\right)$, there exists a unique morphism $u$ with $M_{2} \stackrel{u}{\rightarrow}\left|M_{2}\right|$ such that $q=u f$. Thus $i_{M_{2}} f=f=t_{M_{2}} q=t_{M_{2}} u f$. Since $f$ is an epimorphism, $i_{M_{2}}=t_{M_{2}} u$ and as $i_{M_{2}}$ is a monomorphism, $u$ is a monomorphism. As $\left|M_{2}\right|$ is a free object, by Corollary 3.9, $M_{2}$ is a free object. 


\section{REFERENCES}

[1] H. Crapo, Constructions in combinatorial geometries, Tech. report, Bowdoin College Notes, 1971, N.S.F. Advanced Seminar.

[2] H. Herrlich and G. E. Strecker, Category Theory: An Introduction, Allyn and Bacon Series in Advanced Mathematics, Allyn and Bacon, Massachusetts, 1973. MR 50\#2284. Zbl 265.18001.

[3] J. G. Oxley, Matroid Theory, Oxford Science Publications, Oxford University Press, New York, 1992. MR 94d:05033. Zbl 784.05002.

Talal Ali Al-Hawary: Department of Mathematics, Mu'tah University, P. O. Box 6 Karak, Jordan

E-mail address: ta 1a1@mutah. edu. jo 


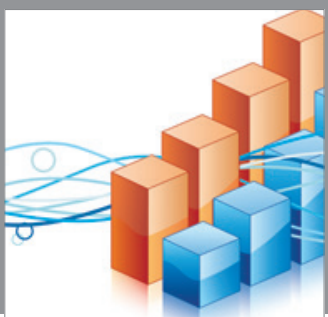

Advances in

Operations Research

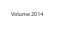

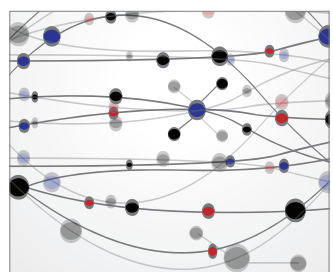

\section{The Scientific} World Journal
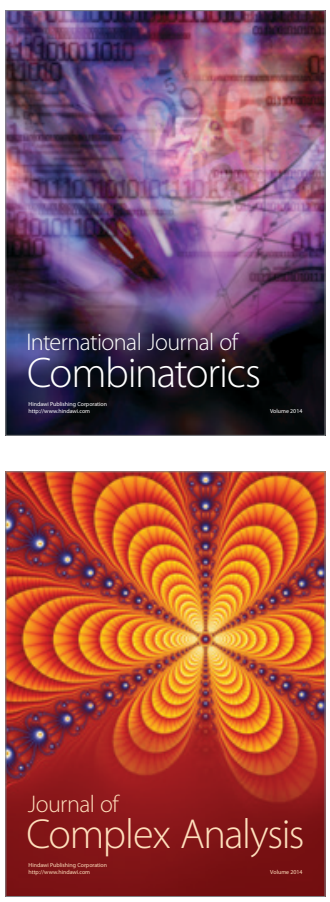

International Journal of

Mathematics and

Mathematical

Sciences
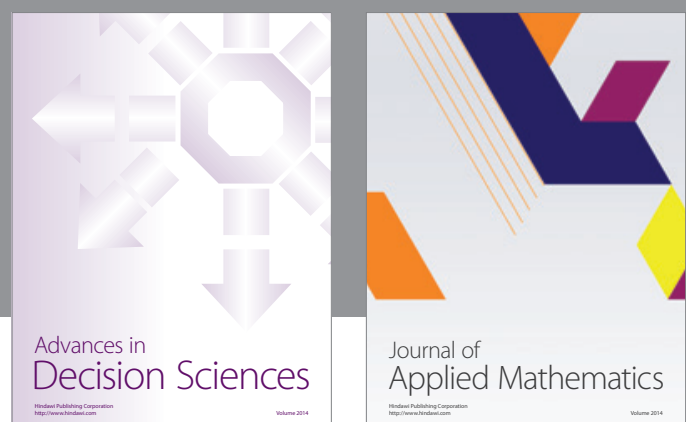

Journal of

Applied Mathematics
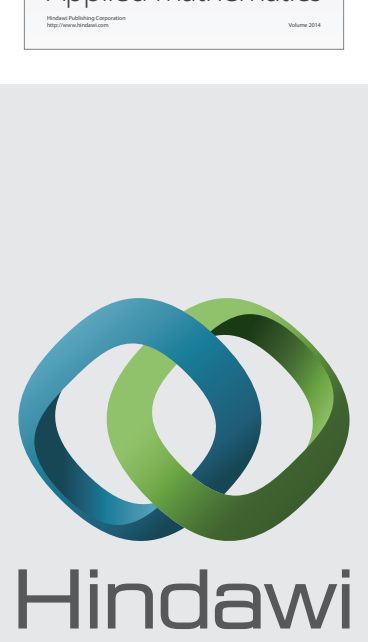

Submit your manuscripts at http://www.hindawi.com
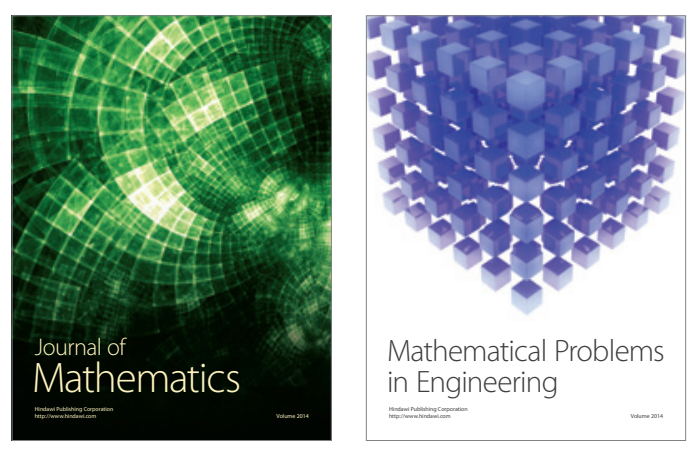

Mathematical Problems in Engineering
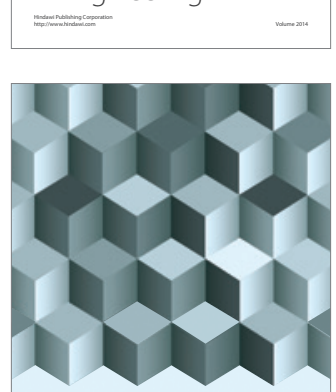

Journal of

Function Spaces
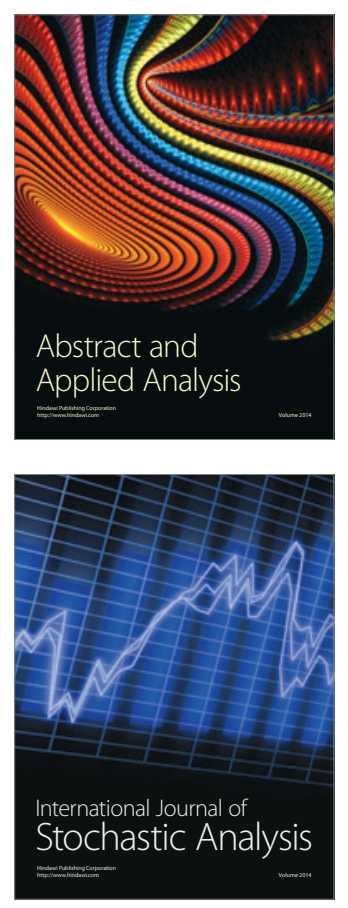

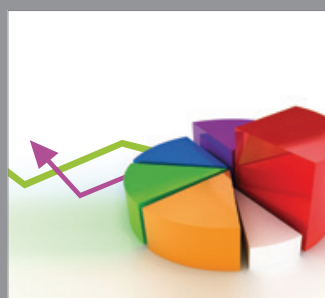

ournal of

Probability and Statistics

Promensencen
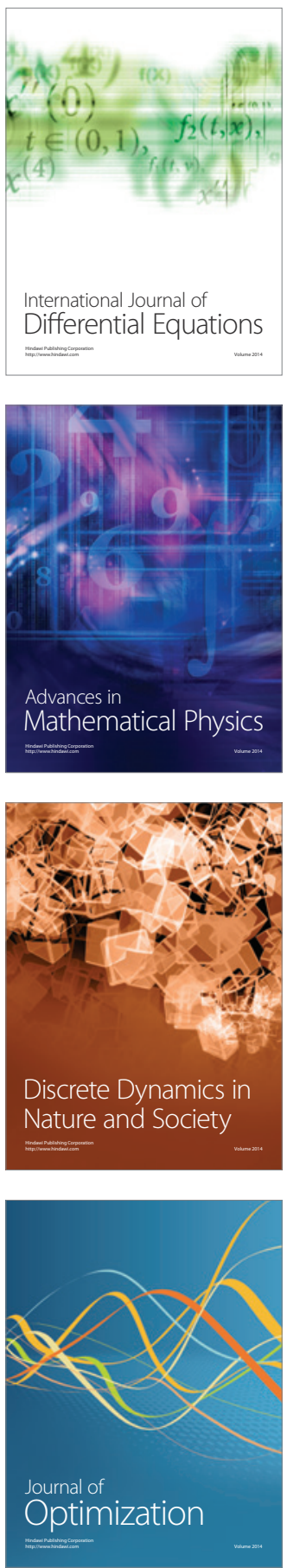